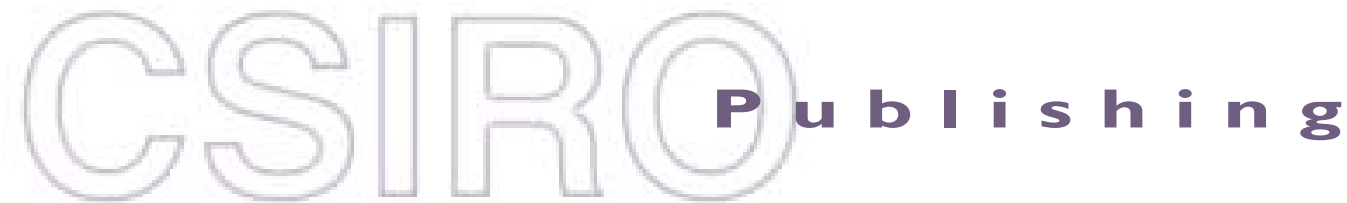

\section{Publications of the Astronomical Society of Australia}

Volume 19, 2002

(C) Astronomical Society of Australia 2002

An international journal of astronomy and astrophysics

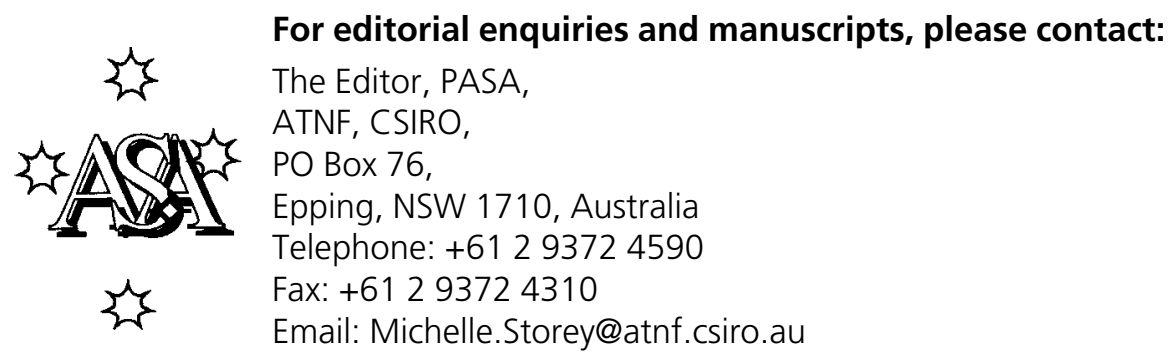

For general enquiries and subscriptions, please contact: CSIRO Publishing PO Box 1139 (150 Oxford St)

Collingwood, Vic. 3066, Australia

Telephone: +6139662 7666

Fax: +61 396627555

Email: publishing.pasa@csiro.au

C S I RO

PUBLISHING Published by CSIRO Publishing

for the Astronomical Society of Australia

www.publish.csiro.au/journals/pasa 


\title{
The Role of Interstellar Scintillation in Intraday Variations at Centimetre Wavelengths
}

\author{
Barney Rickett \\ University of California San Diego, La Jolla, CA 92093, USA \\ bjrickett@ucsd.edu \\ Received 2001 September 3, accepted 2001 November 28
}

\begin{abstract}
The evidence in support of interstellar scintillation as the predominant cause of intraday variations at centimetre wavelengths is reviewed. A new evaluation of the highest brightness temperature implied for AGN is found to be consistent with the Doppler factors in typical jet models, but requires the scattering to occur within about $30 \mathrm{pc}$ of the Earth.
\end{abstract}

Keywords: galaxies: nuclei — galaxies: jets — radio continuum: galaxies — scattering — ISM: general

\section{Introduction to Interstellar Scintillation}

Interstellar scintillation (ISS) causes many well-studied phenomena in the radio observations of pulsars. These include angular and temporal broadening and intensity fluctuations (scintillation) on timescales ranging from minutes to months (see reviews by Rickett 1990 and Narayan 1992). Observations of these phenomena have yielded information on the inhomogeneous electron density in the ionised interstellar medium (ISM) - see Armstrong, Rickett, \& Spangler (1995) and Taylor \& Cordes (1993, hereafter TC93). The latter authors have synthesised a model for the distribution of the interstellar electron density as a sum of contributions from a disk, from spiral arms and the inner Galaxy. In this paper I apply the results to ISS of compact extragalactic sources such as found in active galactic nuclei (AGN). Whereas the majority of extragalactic radio sources have diameters too large to scintillate, some AGN are sufficiently compact to scintillate at a low level. Indeed I will argue that ISS is certainly the dominant and possibly the sole cause of intraday variations (IDV) at centimetre wavelengths.

\subsection{Basic Theory of Scintillation}

Consider waves from a point source that pass through a thin region of inhomogeneous plasma density (a phase screen). The phase perturbations scatter the waves into an angular spectrum of width $\theta_{\text {scatt }}$, typically milliarcseconds (mas) at the frequencies of radio astronomy. A critical scale that governs the scattering is the field coherence scale $s_{d}=1 /\left(k \theta_{\text {scatt }}\right)$ where $k$ is the radio wavenumber; $s_{d}$ is the transverse scale over which there is an rms difference in phase of $1 \mathrm{rad}$, which can be defined from the structure function of phase, calculated on a straight line from the source to the observer. Thus it describes not only a thin plasma screen but also any accumulated phase deviations along the line of sight.

At a distance $L$ from the scattering plasma the mutual interference between components in the angular spectrum converts the phase modulation to an amplitude modulation (i.e. scintillation). This ISS remains weak ( $r m s$ intensity $<$ mean) when the medium introduces a phase change $<1$ rad across a Fresnel zone. This can be written as $s_{d}>r_{f}$, where $r_{f}=\sqrt{L / k}$ defines the Fresnel scale. Thus the ISS is weak near the scattering medium and at high frequencies (distances $\lesssim 500 \mathrm{pc}$ and frequencies above about $3 \mathrm{GHz}$ ). Then the Fresnel scale sets the temporal scale for a point source by

$$
t_{\text {weak }} \sim r_{f} / V=\sqrt{L / k} / V,
$$

where $V$ is the velocity of the ISS pattern past the observer. For a screen at distance $L$ the formulae are precise, but more generally $L$ is the distance to the region of greatest scattering along the line of sight, and the formulae are only approximate.

Strong scintillations occur at the other extreme when $s_{d}<r_{f}$, in which case the diffraction pattern develops two scales referred to as diffractive and refractive. The smaller diffractive scale $\left(s_{d}\right)$ results from interference between components of the angular spectrum; the larger refractive scale $\left(s_{r} \sim L \theta_{\text {scatt }}\right)$ corresponds to the transverse size of the scattering disk, the region that influences the observed intensity at any instant. This determines the largest scale that can influence the amplitude, since still larger scales simply give a tilt in the angle of arrival. When mapped into time by the same pattern velocity $V$ the two timescales are

$$
t_{d} \sim 1 /\left(V k \theta_{\text {scatt }}\right), \quad t_{r} \sim L \theta_{\text {scatt }} / V .
$$

In the ISS of pulsars below $1 \mathrm{GHz}$ the diffractive time is of the order of minutes and the refractive time is of the order of days. The relations highlight the important fact that the two scales depend in opposite ways on the scattering angle. As the frequency is increased this angle decreases and the two scales converge, merging into one, the Fresnel scale, and the scintillation becomes weak above a transition frequency $f_{w}$. Strong scattering phenomena are particularly evident in pulsars which are effectively point sources, and have large flux density at frequencies below $1 \mathrm{GHz}$ where the scattering is usually strong. 


\section{ISS of Extragalactic Sources}

The influence of ISS on the radio flux density from extragalactic sources is largely suppressed by the smoothing that comes from the superposition of diffraction patterns from independently emitting regions over the core of the AGN. In the context of a screen model, relations can be written down simply for the critical source radius that suppresses each regime of scintillation. The source angular radius projected onto the diffraction pattern (at distance $L$ ) blurs the pattern over a scale $L \theta_{\text {source }}$; when this becomes comparable to the spatial scale of the scintillations they are partially suppressed, and the fluctuation time is determined by $L \theta_{\text {source }} / V$. As a consequence there are effective cutoff diameters for the three regimes of ISS: $\theta_{\text {weak }}=r_{f} / L, \theta_{\text {diss }}=s_{d} / L$, and $\theta_{\text {riss }}=s_{r} / L=\theta_{\text {scatt }}$. When $\theta_{\text {source }}$ exceeds these limits the corresponding scintillations decrease in amplitude as $1 / \theta_{\text {source }}$ and increase in timescale as $\theta_{\text {source }}$.

Even the smallest diameter nucleus of an active galaxy (AGN) has an angular extent greater than that of pulsars and so blurs out the diffractive ISS. However, refractive ISS has a less stringent limit since it comes from larger scale inhomogeneities and so causes several forms of intensity variability. Low frequency variations (LFV) over months (Hunstead 1972) are now recognised to be due to refractive ISS (Rickett 1986). At frequencies above a few $\mathrm{GHz}$ scintillations are typically weak at medium or high Galactic latitudes. Variations at a few $\mathrm{GHz}$ over days (termed 'flicker', Heeschen et al. 1987) and IDV at 5-10 GHz over times shorter than a day (Quirrenbach et al. 1989) have both been convincingly demonstrated to be dominated by weak ISS (Rickett 1990) in several specific cases. The chief focus of this paper is to examine the evidence in support of this view, though some debate continues over whether ISS can account for all of the IDV observed at centimetre wavelengths.

Figure 1 shows a plot of extragalactic source diameter against frequency and typical ISS limits. Whereas pulsars lie below all three limit lines for ISS and show all branches of ISS, AGN only show refractive and weak ISS as indicated. There should be a continuum of variability phenomena between LFV and IDV. The intensity variations over several days at $2.3 \mathrm{GHz}$ as monitored with the Green Bank interferometer (e.g. Fiedler et al. 1994) include many examples of ISS intermediate between LFV and IDV.

\section{Observational Evidence for ISS at Centimetre Wavelengths}

Quasar B0917+624 is one of the best studied IDV sources at centimetre wavelengths and its substantial variability on times of a few hours make it the IDV archetype. Though many published papers propose an intrinsic explanation, only a few provide a quantitative model for what physical process sets the timescale. In one such model, Qian et al. (1991) proposed shock structure on scales of $0.1 \mathrm{pc}$ in a relativistic jet.

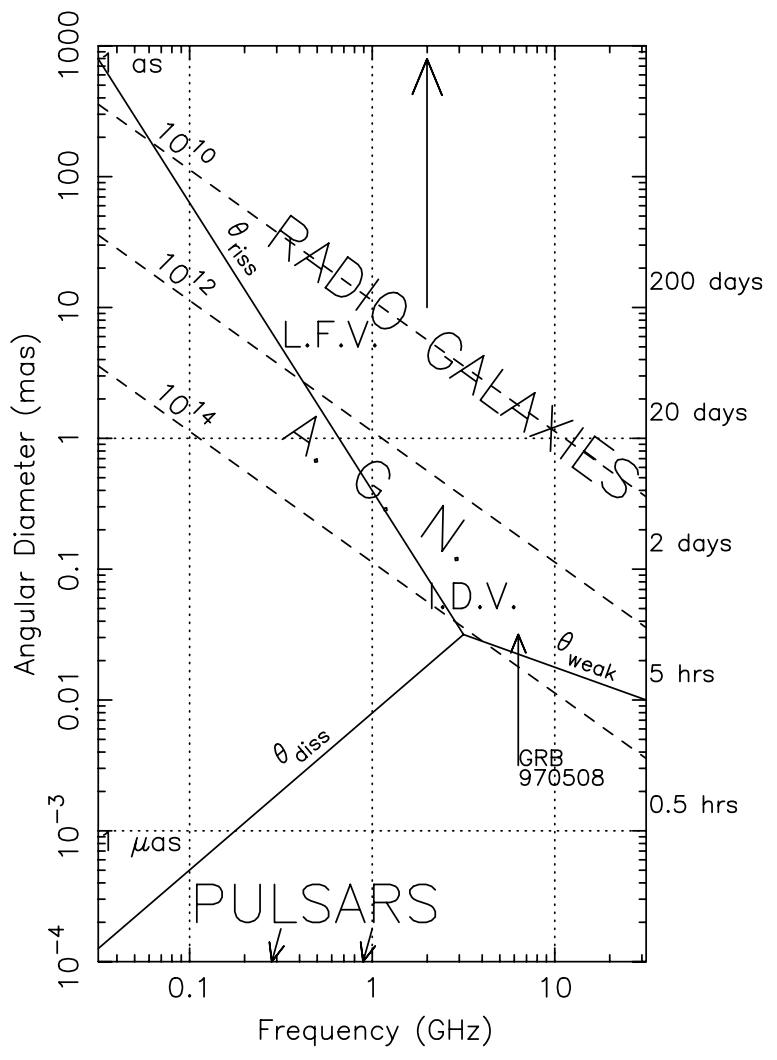

Figure 1 Compact core angular diameters vs frequency. The (solid) limit lines for ISS are shown at Galactic latitude 45 degrees using the TC93 model. For sources that lie above each limit line that branch of ISS is suppressed. Dashed lines are the effective source diameters for sources whose brightness is given by three marked values of $T_{b} / S_{\mathrm{Jy}}$. The times on the right side approximate the timescale for partially quenched ISS at that source diameter. The arrow GRB970508 marks the changing diameter inferred from the decreasing level of ISS at $8.6 \mathrm{GHz}$ by Frail et al. (1997), as the radio afterglow of this gamma ray burst expanded.

A detailed ISS interpretation of the multi-frequency variability in B0917+624 (1988-1990) was proposed by Rickett et al. (1995). A model was presented for the refractive and weak scintillation behaviour, in which the diffractive component was quenched by the source diameter filtering. Model predictions were compared with the observed rms amplitude and characteristic timescale. A good agreement was obtained under two assumptions: (a) the scattering plasma is distributed along the line of sight according to a Gaussian profile with a path length (at $1 / \mathrm{e}$ ) of $200 \mathrm{pc}$; and (b) the effective source diameter is proportional to wavelength, in the fashion expected if the emission is brightness temperature limited in an inhomogeneous synchrotron source. The brightness temperature was estimated to be near $6 \times 10^{12} \mathrm{~K}$, which is compatible with values of the Doppler boosting factor typical for 'super-luminal' sources. Further, they showed that in B0917+624 the $6 \mathrm{~cm}$ variations were closely correlated with a smoothed version of the (simultaneously observed) $3.6 \mathrm{~cm}$ variations; a similar relation was also seen between $6 \mathrm{~cm}$ and $11 \mathrm{~cm}$. This feature of the IDV is entirely consistent with ISS, in which the timescale is 


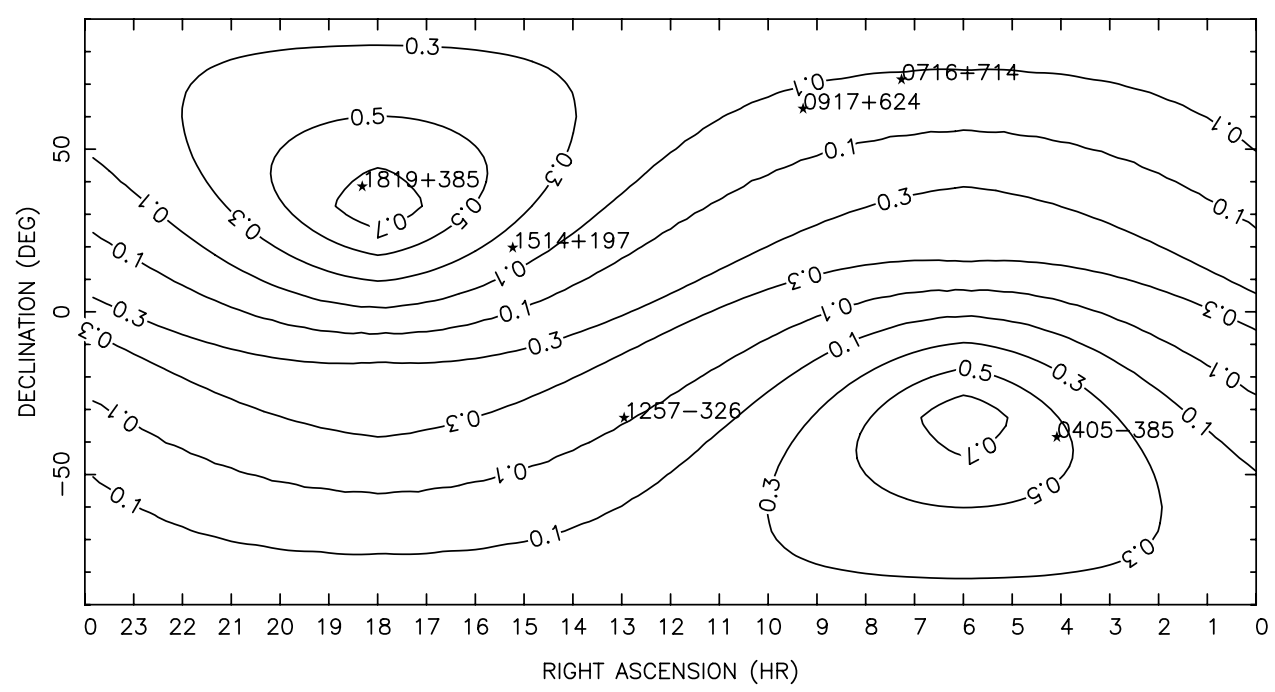

Figure 2 Contours of the ratio between the fastest and slowest ISS timescale in a year predicted if the plasma moves with the LSR; several known IDV sources are marked. The smallest ratio corresponds to the greatest annual modulation; thus the strong annual modulation in $1819+385$ is unexpected and requires plasma moving relative to the LSR.

determined by $L \theta_{\text {source }} / V$ where $\theta_{\text {source }}$ increases linearly with wavelength as noted above. However, the close correlation observed puts a tight limit that the centroid of the $3.6 \mathrm{~cm}$ and $11 \mathrm{~cm}$ emission can be offset by no more than about $10 \mu$ as along the direction of the velocity of the scattering medium relative to the Earth. An analysis of the $20 \mathrm{~cm}$ polarisation variations by Qian et al. (2001) also concluded that refractive ISS is responsible, consistent with a transition frequency near $2.0 \mathrm{GHz}$ and a source Lorentz factor $\gtrsim 8$.

Independent convincing evidence for ISS as the cause of the IDV in J1819+385 comes from the remarkable annual modulation in its timescale (Dennett-Thorpe $\&$ de Bruyn 2001). Such annual modulation can only be caused by apparent rather than intrinsic variations. Evidence for annual modulation in the timescale for B0917+624 was recognised independently by Jauncey \& Macquart (2001) and Rickett et al. (2001). However, Fuhrmann et al. (2002) have failed to confirm the annual modulation, reporting that the IDV has remained slow (or absent) from September 2000 to March 2001. The origin of the extended period of slow variations is not yet clear, though it must reflect changes in the source and/or scattering medium. Figure 2 shows the expected magnitude of the annual modulation ratio as a function of equatorial source coordinates for plasma in the LSR. These predictions will be tested by new IDV observations (e.g. Bignall et al. 2002).

The most rapidly variable $\mathrm{cm}$-wave flux densities are observed from the quasars B0405-385 and J1819+385. In both cases an intrinsic intepretation would imply apparent brightness temperatures as high as $10^{21} \mathrm{~K}$ and hence require Doppler factors of 1000 , far above those needed to explain the superluminal phenomena in other AGN. In both cases the observers (Kedziora-Chudczer et al. 1997; Dennett-Thorpe \& de Bruyn 2000) conclude that ISS is responsible rather than an intrinsic mechanism. These investigators estimate the source size needed to explain the short timescale of the variations. Their results require brightness temperatures in the neighbourhood of $10^{14}-10^{15} \mathrm{~K}$. While this is far below $10^{21} \mathrm{~K}$, it still requires a Doppler factor of 100-1000 (since the relation between the angular diameter inferred from ISS varies only linearly with Doppler factor, instead of as its third power). Thus there remains a difficulty in understanding the source physics even with an ISS explanation for IDV. I now re-examine how ISS can be used to infer the source angular diameter and conclude that these peak brightness temperatures (and Doppler factors) can be reduced by a factor of about 10 .

\section{A Model for ISS of Quasar B0405-385}

I consider the IDV of B0405-385 during its outburst of rapid variations as observed in June 1996 (KedzioraChudczer et al. 1997). As these investigators conclude, I assume that at frequencies of 4.8 and $8.6 \mathrm{GHz}$ it is in the weak ISS regime; hence an explicit model of the scintillation behaviour can be found using the 'Born' approximation, that single scattering in each layer of the medium can be summed linearly to give the power spectrum of intensity fluctuations. This is described by the following equations.

For a plasma layer $z_{o}$ to $z_{o}+\delta z_{o}$ with a given wavenumber spectrum of electron density, we can write the contribution to the scintillation power spectrum as

$$
\begin{aligned}
\delta M\left(q_{x}, q_{y}, z_{o}\right)= & 8 \pi r_{e}^{2} \lambda^{2} P_{N e}\left(q_{x}, q_{y}, z_{o}\right) \delta z_{o} \\
& \times \sin ^{2}\left(\frac{q^{2} z_{o}}{2 k}\right)\left|V\left(\frac{q_{x} z_{o}}{2 \pi}, \frac{q_{y} z_{o}}{2 \pi}\right)\right|^{2}
\end{aligned}
$$

where $P_{N e}\left(q_{x}, q_{y}, z_{o}\right)$ is the 'density spectrum' (i.e. power spectrum of the electron density at wavenumber $\left.\left(q_{x}, q_{y}, q_{z}=0\right)\right)$ at distance $z_{o}$ from the observer and $V(\boldsymbol{u})$ is the source visibilty function at baseline $\boldsymbol{u}$, which 
is normalised by the observing wavelength $\lambda=2 \pi / k$. Whereas the $|V(\boldsymbol{u})|^{2}$ term acts a low-pass filter, the $\sin ^{2}$ term, which is referred to as the Fresnel filter, acts as a high-pass filter. Thus these two filters select out a range of wavenumbers in the medium that contribute most strongly to the intensity spectrum. It is important to note that the central wavenumber is determined by the layer distance $z_{o}$ as well as the source diameter.

Now consider the source brightness function to be a circular Gaussian with diameter at half maximum $\theta_{\text {source }}$; the unit flux visibility is $\exp \left[-3.6\left(\theta_{\text {source }} u\right)^{2}\right]$. I assume a Kolmogorov density spectrum with axial ratio $R$

$$
P_{N e}\left(q_{x}, q_{y}, z_{o}\right)=C_{N}^{2}\left(z_{o}\right)\left(R q_{x}^{2}+q_{y}^{2} / R\right)^{-11 / 6} .
$$

The spatial correlation of intensity is the two dimensional Fourier transform of $\delta M\left(q_{x}, q_{y}, z_{o}\right)$ for each layer $\delta z_{o}$ in thickness. In the Born approximation we sum over $\delta z_{o}$ for an extended scattering medium. The autocorrelation of intensity at a single antenna, moving at velocity $V_{\text {eff }}$ relative to the scintillation pattern, can be written

$$
R_{I I}^{t}(\tau)=\int d^{2} \boldsymbol{q} \delta M(\boldsymbol{q}) \exp \left[-j \boldsymbol{q} \cdot \boldsymbol{V}_{\mathrm{eff}} \tau\right] .
$$

The zero lag value of this function is the variance of the intensity and we define a characteristic timescale as the time lag $\tau_{w}$ where $R$ falls to half of $R_{I I}^{t}(0)$. These two quantities have also been estimated from the observations. Thus we have a set of expressions from which the scintillation index $m=\sqrt{R_{I I}^{t}(0)}$ and timescale can be computed. The controlling parameters are the source diameter, the medium distance, the plasma anisotropy (axial ratio) and its orientation with respect to the velocity, and the velocity. With the recognition of annual modulation effects in ISS we are now reasonably confident in estimating the velocity as the Earth's velocity relative to the local standard of rest $\left(36 \mathrm{~km} \mathrm{~s}^{-1}\right.$ for the observations in June 1996).

Figure 3 shows the normalised autocorrelation function observed at $8.6 \mathrm{GHz}$. The $1 \sigma$ statistical error as indicated is substantial and does not decrease at large lags. Thus while the minimum at $1.25 \mathrm{hr}$ is $4 \sigma$ from zero and so requires an explanation, the maximum at $2.6 \mathrm{hr}$ and the minimum at $3.8 \mathrm{hr}$ are within $2 \sigma$ of zero and are presumed to be statistical errors. Theoretical curves are overplotted assuming a screen at $25 \mathrm{pc}$ and $\theta_{\text {source }}=15 \mu$ as. Calculations for four axial ratios are plotted, where an axial ratio 2.0 is oriented with the velocity vector along the major axis and for axial ratios $<1.0$ it is along the minor axis. Only axial ratios of 0.5 and 0.25 come within $2 \sigma$ of matching the observed negative 'overshoot' at $1.25 \mathrm{hr}$. These calculations will be presented in greater detail in a paper in preparation, discussing the effects of even smaller axial ratios, changing the screen distance, extended scattering and source size. It is shown that for axial ratios smaller than 0.25 the minimum correlation overshoot saturates at -0.48 . In the light of the errors, we conclude that the plasma must have a substantial anisotropy and adopt an axial ratio of 0.25 . The calculations also show that the negative overshoot is

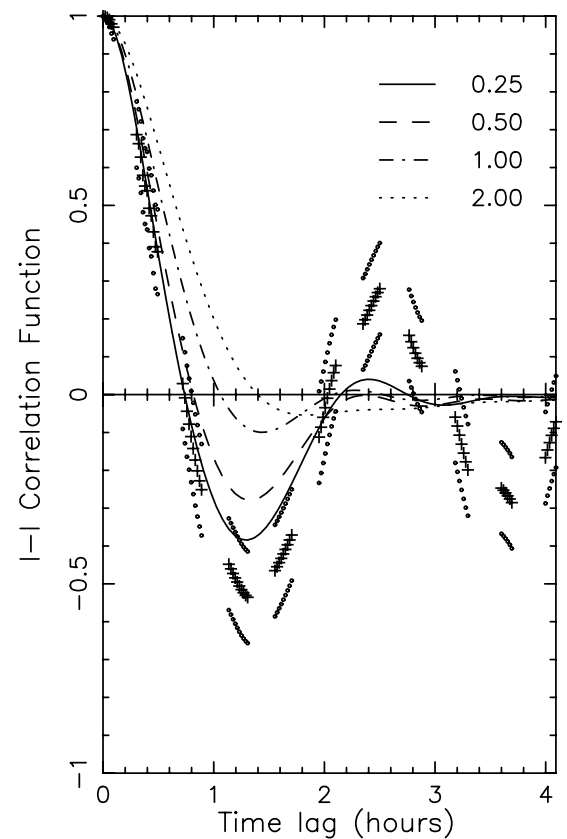

Figure 3 The autocorrelation function $(+)$ and their $1 \sigma$ errors (dots) for $8.6 \mathrm{GHz}$ IDV of quasar B0405-385. Four theory lines with axial ratios indicated assume weak ISS in a screen at $25 \mathrm{pc}$ for a Gaussian source of $15 \mu$ as diameter. The data require an axial ratio $\lesssim 0.25$, corresponding to the velocity vector being transverse to the plasma filamentary structure.

visible over a range \pm 45 degrees in the orientation. Thus the overshoot phenomenon should be relatively common.

The overshoot can also be viewed as quantifying the quasi-periodic nature of the IDV. As mentioned above, there is a bandpass in the wavenumber filter which, when the velocity is oriented along the wide direction of the wavenumber spectrum, leads to a peak in the temporal spectrum and quasi-periodic fluctuations. It is worth emphasising that an anisotropic source visibility cannot duplicate this effect, in which the observed intensity spectrum dips at low frequencies. The magnitude of any visibility function is greatest at zero baseline (equal total source flux density) and so multiplication by $|V|^{2}$ cannot cause a low frequency dip. However, the source diameter strongly controls the timescale and if large enough could suppress the oscillatory behaviour. The quasi-periodic nature of the fluctuations has been noted in the context of intrinsic explanations for IDV; but in no case has there been a quantitative comparison of theory with observations such as in Figure 3.

Figure 4 shows the trade-off between screen distance and source diameter in matching the observed scintillation index $m$ (rms variation in flux density normalised by the mean for the compact core) and timescale $\tau_{w}$. With velocity and anisotropy fixed as noted, the equations above were used to compute $m$ and $\tau_{w}$ numerically for a grid of points versus scattering distance $\left(z_{o}\right)$ and source diameter. Pairs of contours mark acceptable limits to match the observed $m$ and $\tau_{w}$. This calculation includes the possibility that only a fraction of the total flux density is in the 


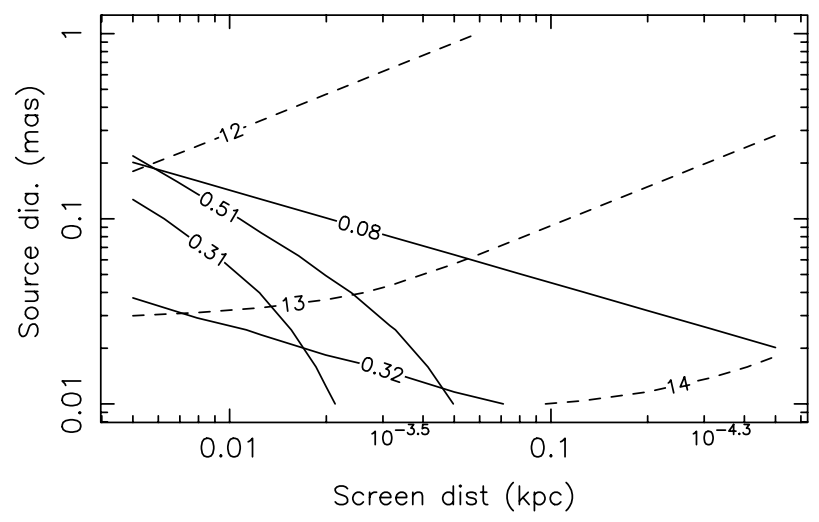

Figure 4 Contours of $m$ and $\tau_{w}$ vs $z_{o}, \theta_{\text {source }}$; pairs of contours are marked with the upper and lower bounds observed at $8.6 \mathrm{GHz}$ for B0405-385 $\left(0.08<m<0.32,0.3<\tau_{w}<0.5 \mathrm{hr}\right)$; dashed lines show the $\log _{10}$ of the implied source brightness temperature. Two values of the scattering measure of the screen (in $\mathrm{m}^{-20 / 3} \mathrm{kpc}$ ) are given along the distance axis.

scintillating compact core; the lower bound for $m$ corresponds to all of the source scintillating; the upper bound is derived from the minimum observed flux density. Consequently at each point in the $\left(z_{o}, \theta_{\text {source }}\right)$ plane we can calculate the associated compact flux density and diameter and hence the source brightness temperature, which is given by the dashed contours. The conclusion is that in the range of allowable source models the brightness temperature $\lesssim 3 \times 10^{13} \mathrm{~K}$. Whereas this resolves the brightness problem for B0405-385 it requires scattering concentrated remarkably near the Sun (in the range 5-30 pc). This creates a puzzle for our understanding of the irregular plasma in the ISM, for which the TC93 model gives a path length of 500-1000 pc in this direction.

These calculations were done for a screen model; they have also been done for an extended medium with turbulence parameter $C_{N}^{2}\left(z_{o}\right)$ following a Gaussian profile with path length $z_{g}$ at 1/e. Plots similar to Figure 3 are shown by B. Rickett et al. (in preparation) in which the horizontal axis is $z_{g}$; the results are similar but allow values of $z_{g}$ about two times greater than for the screen model.

\section{Discussion}

There is now strong evidence that ISS explains the two most rapid IDV sources and also the archetype IDV source B0917+624. Further, as argued above, the brightness temperatures needed are comparable to those implied by superluminal component motion and so are compatible with 'standard' jet models. However, I note that the resolution of the brightness problem has come at the expense of introducing an ISM problem, leading to the need to improve our understanding of our local plasma environment.

Though these are only particular examples and so do not necessarily imply that all centimetre wavelength IDV are due to ISS, they raise that possibility. I have presented evidence that the detailed observational signatures of several particular examples of IDV can be explained by ISS without recourse to the additional assumption of intrinsic variations on timescales shorter than $12 \mathrm{hr}$. It is then natural to ask what evidence remains in support of intrinsic IDV at these wavelengths. The most cited evidence is from the optical and radio monitoring of quasar B0716+714. Quirrenbach et al. (1991) showed remarkable similarities in the signature of the IDV light curves at $6 \mathrm{~cm}$ and optical R-band over a monitoring period of about 6 days. The variations are not formally correlated (the cross-correlation being negative at zero time offset). However, as noted by Jauncey (2002), the simultaneous observations at $2 \mathrm{~cm}$, $3.6 \mathrm{~cm}$, and $6 \mathrm{~cm}$ (Quirrenbach et al. 2000) showed quite substantial differences among the radio bands (in general agreement with the wavelength dependence of the IDV timescale noted above for B0917+624). This seems to weaken the view that the IDV is an ultra-broadband phenomenon since intermediate frequencies exhibit rather different light curves. Of course it does not affect the argument for an intrinsic cause for the optical fluctuations which cannot be due to ISS, but which do not imply a brightness problem. Gabuzda (2002) argued that changes in polarised AGN structure observed on times of 6-12 hr are evidence for intrinsic variability (see also Gabuzda et al. 1989, 2000a,b); however, in such cases the implied Doppler factors are less extreme. Krichbaum et al. (2002) argue for the simultaneous influence of intrinsic IDV and ISS in total flux density, particularly at the relatively longer timescales of 6-24 hr. However, the extremely compact source diameters implied by any such intrinsic variation must also be accompanied by rapid ISS. Thus a consensus seems to exist that ISS plays a central role in centimetre wavelength IDV.

\section{Acknowledgments}

I thank Bill Coles for valuable discussions; I thank Lucyna Kedziora-Chudczer, David Jauncey, Ger de Bruyn, Thomas Krichbaum, and Arno Witzel for sharing their observations with me. I thank the US NSF for support under grant AST 9988398. I thank Ron Ekers, the Director, for providing support for my visit to the ATNF.

\section{References}

Armstrong, J. W., Rickett, B. J., \& Spangler, S. R. 1995, ApJ, 443, 209

Bignall, H. E., et al. 2002, PASA, 19, 23

Dennett-Thorpe, J., \& de Bruyn, A. G. 2000, ApJ, 529, L65

Dennett-Thorpe, J., \& de Bruyn, A. G. 2001, Ap\&SS, 278, 101

Fiedler, R., Dennison, B., Johnston, K., Waltman, E., \& Simon, R. 1994, ApJ, 430, 581

Frail, D. A., Kulkarni, S. R., Nicastro, L., Feroci, M., \& Taylor, G. B. 1997, Nature, 389, 261

Fuhrmann, L., et al. 2002, PASA, 19, 64

Gabuzda, D. C. 2002, PASA, 19, in press

Gabuzda, D. C., Wardle, J. F. C., \& Roberts, D. H. 1989, ApJ, 338,743

Gabuzda, D. C., Kochenov, P. Yu., Cawthorne, T. V., \& Kollgard, R. I. 2000a, MNRAS, 313, 627

Gabuzda, D. C., Kochenov, P. Yu., \& Cawthorne, T. V. 2000b, MNRAS, 319, 1125 
Heeschen, D. S., Krichbaum, T. P., Schalinski, C. A., \& Witzel, A. 1987, AJ, 94, 1493

Hunstead, R. W. 1972, ApL, 12, 193

Jauncey, D. L. 2002, private communication

Jauncey, D. L., \& Macquart, J.-P. 2001, A\&A, 370, L9

Krichbaum, T. P., Kraus, A., Fuhrmann, L., Cimò, G., \& Witzel, A. 2002, PASA, 19, 14

Kedziora-Chudczer, L., Jauncey, D. L., Wieringa, M. H., Walker, M. A., Nicholson, G. D., Reynolds, J. E., \& Tzioumis, A. K. 1997, ApJ, 490, L9

Narayan, R. 1992, Phil. Trans. R. Soc. London A, 341, 151

Qian, S. J., Quirrenbach, A., Witzel, A., Krichbaum, T. P., Hummel, C. A., \& Zensus, A. 1991, A\&A, 241, 15
Qian, S. J., Witzel, A., Kraus, A., Krichbaum, T. P., \& Zensus, A. 2001, A\&A, 367, 770

Quirrenbach, A., Witzel, A., Krichbaum, T. P., Hummel, C. A., Alberdi, A., \& Schalinski, C. J. 1989, Nature, 337, 442

Quirrenbach, A., et al. 1991, ApJ, 372, L71

Quirrenbach, A., et al. 2000, A\&AS, 141, 2212

Rickett, B. J. 1986, ApJ, 307, 564

Rickett, B. J. 1990, ARA\&A, 28, 561

Rickett, B. J., Quirrenbach, A., Wegner, R., Krichbaum, T. P., \& Witzel, A. 1995, A\&A, 293, 479

Rickett, B. J., Witzel, A., Kraus, A., Krichbaum, T. P., \& Qian, S. J. 2001, ApJ, 550, L11

Taylor, J. H., \& Cordes, J. M. 1993, ApJ, 411, 674 (TC93) 